Descripción de cultivar

\title{
Agrosavia La 22: primera variedad de arracacha en Colombia
}

\author{
Johanna Paola Garnica Montaña ${ }^{1 \S}$ \\ Liliana Margarita Atencio Solano ${ }^{2}$ \\ Oscar Jair Rodríguez Rodríguez ${ }^{3}$ \\ Jorge Enrique Villamil Carvajal ${ }^{1}$
}

${ }^{1}$ Corporación Colombiana de Investigación Agropecuaria-AGROSAVIA-Centro de Investigación Nataima. Vía Espinal-Ibagué km 9, Tolima, Colombia. CP. 733529. (jgarnicam@agrosavia.co; jvillamil@agrosavia.co). ${ }^{2}$ AGROSAVIA-Centro de Investigación Turipana. Vía Montería-Cereté km 13, Córdoba, Colombia. CP. 230550. (latencio@agrosavia.co). ${ }^{3}$ Universidad Estatal de Dakota del Norte. Dakota del Norte, EE. UU. CP. 58105. (oscar.rodriguez@ndsu.edu).

${ }^{\S}$ Autora para correspondencia: jgarnicam@agrosavia.co.

\section{Resumen}

En Colombia se cultivan alrededor de siete materiales regionales en monocultivo y ocasionalmente en asocio, el ciclo es anual y sin estacionalidad de siembra, lo que permite cosechar durante todo el año. La producción es baja con rendimientos que varían entre 7 y 13 t ha ${ }^{-1}$ y se presentan problemas fitosanitarios sumado a la presencia de raíces comerciales con pigmentaciones púrpuras no deseadas en la comercialización. Con el propósito de ofertar nuevos genotipos, incrementar los rendimientos y atender la demanda de raíces tuberosas amarillas, se obtiene la variedad Agrosavia La 22 que conserva el color de la raíz y es el primer genotipo de esta especie en ser inscrito en el registro nacional de cultivares en Colombia. Agrosavia La 22 es el resultado de la selección de plantas provenientes de semilla sexual que se generaron luego del proceso de polinización cruzada de accesiones conservadas en el banco de germoplasma en Colombia. Se utilizaron descriptores cualitativos y cuantitativos de planta completa, hoja, semilla, tallo y raíz tuberosa, además de variables de rendimiento. Luego de cinco ciclos de evaluación los resultados muestran que esta variedad tiene raíces completamente amarillas, rendimientos experimentales de raíces comerciales de $33.48 \mathrm{t} \mathrm{ha}^{-1}$ y no comerciales de $1.31 \mathrm{t} \mathrm{ha}^{-1}$. En promedio una planta tiene nueve raíces comerciales cada una con un peso promedio de $271 \mathrm{~g}, 15$ propágulos por planta y tolerancia a plagas y enfermedades frente a los genotipos usualmente usados.

Palabras clave: Arracacia xanthorrhiza, germoplasma, raíces tuberosas, rendimiento, raíz amarilla.

Recibido: enero de 2021

Aceptado: febrero de 2021 
La arracacha 'Arracacia xanthorrhiza Bancroft' pertenece a la familia Apiaceae, se considera como la única umbelífera domesticada en Suramérica (Hermann, 1997) y la región andina se precisa como su centro de origen (Rueda, 1992). Se siembra principalmente en países de Suramérica y es conocida como arracacha, apio, zanahoria blanca o racachu en diferentes países de la región andina y mandioquinha-salsa en Brasil (Dos Santos, 2004). Se ha reportado la mayor diversidad genética en especies del género Arracacia en Colombia donde la conservación ha sido favorecida por diversas etnias y culturas indígenas que la cultivaban para venta o auto consumo (Vasquez et al., 2004; Madeira y Ferreira, 2016).

Algunos autores la describen como una planta perenne, pero otros la consideran anual atendiendo al aprovechamiento en la producción de raíces tuberosas (Garnica-Montaña et al., 2021). Generalmente, el ciclo de producción se encuentra entre 10 y 14 meses dependiendo de la zona altitudinal en un rango de 400 a $3600 \mathrm{msnm}$, la altitud ideal en el trópico es de 1500 a $2500 \mathrm{~m}$ (Hermann, 1997). La planta de A. xanthorrhiza se compone de cuatro partes: la parte aérea comprende hojas compuestas y los propágulos o semillas asexuales adheridas a un tallo modificado llamado cepa o corona y en la parte subterránea se desarrollan las raíces tuberosas que presentan coloraciones generalmente blancas, amarillas, naranjas y púrpura.

Aunque todos los órganos se aprovechan, ésta especie se cultiva principalmente por las raíces tuberosas utilizadas en la alimentación humana las cuales, tienen un alto valor nutricional debido a sus contenidos de proteínas, nutrientes como $\mathrm{Ca}, \mathrm{P}$ y $\mathrm{Fe}$ y vitaminas $\mathrm{A}, \mathrm{B}$ y $\mathrm{C}$, son de fácil digestibilidad, aportan energía asociada a los altos contenidos de almidón (25\%) y se consideran un alimento funcional (Leidi et al., 2018; Pinto-Acero et al., 2019). En Suramérica tradicionalmente se utilizan en la elaboración de sopas, estofados, guisos, pures, panes, galletas y bebidas (Albano et al., 2014).

La reproducción sexual es escasa y dependiente de condiciones ambientales y el genotipo (Knudsen, 2003). Bell y Constance (1966) reportaron que A. xanthorrhiza es diploide con conformación $2 \mathrm{n}=2 \mathrm{x}=44$ número base $\mathrm{n}=22$, esta condición también se presentó en la especie silvestre Tauschia con la que está estrechamente relacionada. Por otra parte, Morillo et al. (2004) propuso la especie como tetraploide con conformación $2 n=4 x=44$ y con número base $n=11$ según el recuento cromosómico y el cariotipo, manifestando que 'es una especie tetraploide posiblemente diploidizada, por lo que recomienda analizar los datos como un verdadero diploide’.

A nivel mundial, Colombia se posiciona como el segundo productor después de Brasil, reportando para 2018 un total de 9653 ha sembradas en 15 departamentos con un rendimiento promedio de $9.3 \mathrm{t} \mathrm{ha}^{-1}$ y una producción de $110058 \mathrm{t}$; sin embargo, cerca de $60 \%$ se concentra en el departamento del Tolima (MinTIC, 2018). El sistema productivo tiene importancia agroalimentaria y socio económica, en muchas regiones se siembra entre 0.5 a 20 ha aproximadamente, asociado a la mano de obra familiar y diversos sistemas productivos (maíz, arveja, frijol y otros), es considerado un cultivo de seguridad alimentaria (Alvarado y Ochoa, 2010).

Aunque en Colombia existe una importante diversidad genética, una de las principales limitantes en la producción es la baja disponibilidad de genotipos comerciales. Muñoz et al. (2015) reportan que el cultivar regional 'amarilla común' representó $80 \%$ del área sembrada en Boyacá y 95\% en el Tolima, departamento con las mayores áreas de siembra. 
Este genotipo presenta rendimientos de $10 \mathrm{t} \mathrm{ha}^{-1}$, pigmentación púrpura de raíces en $10 \%$, enfermedades del follaje ocasionadas por los hongos Colletotrichum sp., Alternaria sp. y Cercospora sp., e insectos plaga como trips y áfidos, factores que son considerados limitantes para los productores y comercializadores, atendiendo a las exigencias del mercado basadas en la demanda principalmente de raíces tuberosas amarillas para consumo en fresco.

Para dar respuesta a estas limitantes, durante el año 2013 AGROSAVIA inició investigaciones para obtener una variedad de arracacha de alto rendimiento, bajos costos de producción y raíces completamente amarillas. Es así, como en el año 2019, el instituto Colombiano Agropecuario (ICA) le asigna el ingreso al registro nacional de cultivares a esta nueva variedad.

\section{Origen y selección}

A partir del 2013 la Corporación Colombiana de Investigación Agropecuaria-AGROSAVIA impulsó proyectos de investigación que respondieran a las demandas de los sistemas productivos. En el Tolima se iniciaron investigaciones para la obtención de variedades mejoradas de arracacha que den respuesta a los requerimientos del mercado y a condiciones bióticas y abióticas de la región (MADR, 2019). El proceso de investigación inició con la selección de 21 accesiones pertenecientes al sistema de bancos de germoplasma de la nación para la alimentación y la agricultura (SBGNAA) por medio de multiplicación vegetativa. Adicionalmente, se colectó un material proveniente de semilla sexual, completando de esta forma 22 materiales iniciales. Todos los genotipos fueron seleccionados por presentar la característica de pigmentación amarilla de sus raíces tuberosas.

Luego, se desarrolló un proceso de selección clonal durante diferentes ciclos productivos comprendidos entre los años 2013 y 2015 buscando principalmente materiales con raíces de tonalidad amarilla y alta producción. Como resultado de este proceso, se identificó el genotipo proveniente de semilla sexual como material promisorio, el cual, fue evaluado en el año 2016 donde se comparó con dos materiales regionales sembrados en el país, 'amarilla común' y 'yema de huevo'. Para efectuar las comparaciones entre genotipos se estableció una prueba de evaluación agronómica (PEA) en cinco localidades como requisito para la distribución e ingreso de un genotipo al registro nacional de cultivares del Instituto Colombiano Agropecuario (ICA).

Durante 2017 se estableció la prueba semicomercial en áreas de 0.5 ha en tres localidades para corroborar los resultados obtenidos durante el proceso de selección y evaluación. Con la PEA y la prueba semicomercial se confirmó la estabilidad de la característica de raíces con tonalidad amarilla, adaptabilidad del material y su producción de forma adicional, se confirmaron algunas características como su tolerancia a plagas y enfermedades en comparación a los testigos evaluados. Finalmente, en 2019 el ICA otorgó el registro de Agrosavia La 22 mediante la resolución No. 00015201 para la región natural andina con altitudes entre 1 200-1 800 m a 1 800$2200 \mathrm{~m}$ y mayor a $2200 \mathrm{~m}$. Se resalta que durante el proceso de evaluación los productores mostraron interés por el material y demanda de la semilla de forma constante. Agrosavia La 22, es una variedad producto de la polinización cruzada de accesiones florecidas durante el año 2011.

La planta madre ILS 3915, que pudo actuar también como padre, presenta peciolo verde amarilloso con púrpura rojizo regularmente distribuido y raíz tuberosa amarillo claro, participaron como posibles padres, las accesiones ILS3916 y ILS3917. La accesión ILS 3916 tiene peciolos verde oliva sin coloración secundaria y raíz principal blanca con pigmentaciones violeta y la ILS3917 posee peciolos verde oliva fuerte con rayado tenue púrpura y color de la raíz amarillo claro. 


\section{Adaptación y rendimiento}

Las evaluaciones por adaptación y rendimiento se realizaron en fincas de productores en los municipios de Cajamarca e Ibagué, Tolima, ubicados en la zona andina de Colombia, con una precipitación media anual de $826.4 \mathrm{~mm}$, temperatura media de $18.1^{\circ} \mathrm{C}$, suelos franco-arenosos y zona de vida bmh-PM y bh-MB Corporación Autónoma Regional del Tolima (CORTOLIMA, 2006).

El material promisorio fue colectado como 'segregante', posteriormente paso a ciclos de evaluación y selección en lotes de productores con diferentes nombres. En el año 2013 se establecieron los 22 genotipos a una altura de $1985 \mathrm{msnm}$ y se sembró como 'parcela 22', durante el 2014 se sembraron 10 accesiones seleccionadas por adaptación y producción y se estableció como 'parcela 10' a 1955 msnm y en el año 2015 se evaluaron nuevamente los 10 materiales en condiciones contrastantes a una altura de $2800 \mathrm{msnm}$. En el año 2016 se selecciona por su buen comportamiento y se siembra con el nombre 'clon 22' en una PEA junto con los materiales regionales, yema de huevo y amarilla común, en cinco localidades de Cajamarca e Ibagué con altitudes de 1 796, 2 040, 2 063, 2091 y 2536 m. En 2017, se estableció la prueba semicomercial en un área de 0.5 ha en tres localidades ubicadas a 2 062, 2127 y 2159 msnm. Por la identificación en campo, el conocimiento de productores y la aceptación del nombre en la zona se denominaron Agrosavia La 22.

El Cuadro 1 muestra el comportamiento de Agrosavia La 22 en los cinco ciclos de evaluación mencionados anteriormente. Se registró información de peso de parte aérea que incluye hojas con peciolo, peso de propágulos o semilla asexual y peso de corona o cepa en $\mathrm{kg} \mathrm{planta}^{-1}$, además de número de propágulos y número de raíces comerciales por planta. La variedad mostró un promedio de $0.55 \mathrm{~kg}$ de parte aérea (hoja) por planta, $1.08 \mathrm{~kg}$ de cepa con 16 propágulos que pesan en total $0.32 \mathrm{~kg}$ planta ${ }^{-1}$. Los rendimientos de raíces comerciales oscilaron entre $20 \mathrm{y} 56 \mathrm{t} \mathrm{ha}^{-1}$ y las plantas desarrollaron entre 6 y 14 raíces tuberosas. El rendimiento no comercial se encuentra entre 0.99 y $2.87 \mathrm{t} \mathrm{ha}^{-1}$. En Colombia, se consideran raíces comerciales, aquellas raíces con un longitud y diámetro mayor a $8 \mathrm{~cm}$ y $3 \mathrm{~cm}$, respectivamente (CARC, 2014).

Cuadro 1. Evaluación de Agrosavia La 22 en cinco ciclos de evaluación en diferentes localidades de los municipios de Cajamarca e Ibagué, Tolima, Colombia.

\begin{tabular}{|c|c|c|c|c|c|c|c|c|c|c|}
\hline \multirow{2}{*}{ Año } & \multirow{2}{*}{ Proceso } & \multirow{2}{*}{$\begin{array}{c}\text { Altitud } \\
\text { (m) }\end{array}$} & PAE & PPR & PCE & NPR & NRC & PRC & PRNC & \multirow{2}{*}{ CRT } \\
\hline & & & \multicolumn{3}{|c|}{$\left(\right.$ kg planta $\left.^{-1}\right)$} & \multicolumn{2}{|c|}{ (Núm. planta) } & \multicolumn{2}{|c|}{$\left(\mathrm{t} \mathrm{ha}^{-1}\right)$} & \\
\hline 2013 & 22 genotipos & 1985 & 0.9 & 0.68 & 0.62 & 20 & 14 & 56 & 0 & Amarillo \\
\hline 2014 & 10 genotipos & 1955 & 1.33 & 0.73 & 1.67 & 23 & 7 & 31.32 & 2.19 & Amarillo \\
\hline 2015 & 10 genotipos & 2800 & 0.15 & 0.23 & 1.78 & 15 & 6 & 20.12 & 1.19 & Amarillo \\
\hline \multirow[t]{5}{*}{2016} & \multirow{5}{*}{$\begin{array}{c}\text { PEA (1 genotipo } \\
\text { promisorio, } 2 \\
\text { testigos) }\end{array}$} & 1796 & 0.98 & 0.28 & 1.37 & 16 & 7 & 29.26 & 1.38 & Amarillo \\
\hline & & 2040 & 0.25 & 0.19 & 1.14 & 15 & 12 & 47.12 & 1.28 & Amarillo \\
\hline & & 2063 & 0.42 & 0.21 & 0.91 & 14 & 6 & 20.33 & 1.18 & Amarillo \\
\hline & & 2091 & 0.48 & 0.18 & 0.89 & 12 & 12 & 40.58 & 2.87 & Amarillo \\
\hline & & 2536 & 0.45 & 0.25 & 0.96 & 13 & 10 & 30.43 & 1.07 & Amarillo \\
\hline
\end{tabular}




\begin{tabular}{|c|c|c|c|c|c|c|c|c|c|c|}
\hline \multirow{2}{*}{ Año } & \multirow{2}{*}{ Proceso } & \multirow{2}{*}{$\begin{array}{l}\text { Altitud } \\
\text { (m) }\end{array}$} & PAE & PPR & PCE & NPR & NRC & PRC & PRNC & \multirow{2}{*}{ CRT } \\
\hline & & & \multicolumn{3}{|c|}{$\left(\mathrm{kg} \mathrm{planta}^{-1}\right)$} & \multicolumn{2}{|c|}{ (Núm. planta) } & \multicolumn{2}{|c|}{$\left(\mathrm{t} \mathrm{ha}^{-1}\right)$} & \\
\hline \multirow[t]{3}{*}{2017} & \multirow{3}{*}{$\begin{array}{l}\text { Prueba semi- } \\
\text { comercial }\end{array}$} & 2062 & 0.31 & 0.23 & 0.8 & 14 & 9 & 38.25 & 0.99 & Amarillo \\
\hline & & 2127 & 0.31 & 0.24 & 0.96 & 16 & 11 & 23.75 & 1.09 & Amarillo \\
\hline & & 2159 & 0.47 & 0.29 & 0.84 & 17 & 9 & 31.12 & 1.19 & Amarillo \\
\hline
\end{tabular}

$\mathrm{PAE}=$ peso parte aérea; $\mathrm{PPR}=$ peso de semilla asexual o propágulo; $\mathrm{PCE}=$ peso de corona o cepa; $\mathrm{NPR}=$ número de propágulos; $\mathrm{NRC}=$ número de raíces comerciales; $\mathrm{PRC}=$ peso de raíces comerciales; $\mathrm{PRNC}=$ peso de raíces no comerciales; $\mathrm{CRT}=$ color de raíz tuberosa.

El Cuadro 2 muestra los resultados de la evaluación de la variedad junto con los cultivares regionales amarilla común y yema de huevo, el primero considerado el material comercial en el mercado nacional, pero con la desventaja de presentar raíces tuberosas con pigmentación púrpura y bajo rendimiento (Alvarado y Ochoa, 2010). Los resultados de las PEA permitieron conocer el comportamiento frente a los testigos, donde Agrosavia La 22 se destacó principalmente por presentar los mayores valores en producción de raíces tuberosas comerciales $\left(33.54 \mathrm{tha}^{-1}\right)$ que son completamente amarillas, además el número de propágulos (14 propágulos planta ${ }^{-1}$ ) y raíces comerciales ( 9 raíces planta $^{-1}$ ). Características de gran importancia para el productor porque están directamente asociados al rendimiento y con ello a los ingresos y también a la obtención de semillas para próximos ciclos de producción.

Cuadro 2. Variables de producción de la prueba de evaluación agronómica en cinco localidades de Cajamarca e Ibagué, Tolima, Colombia.

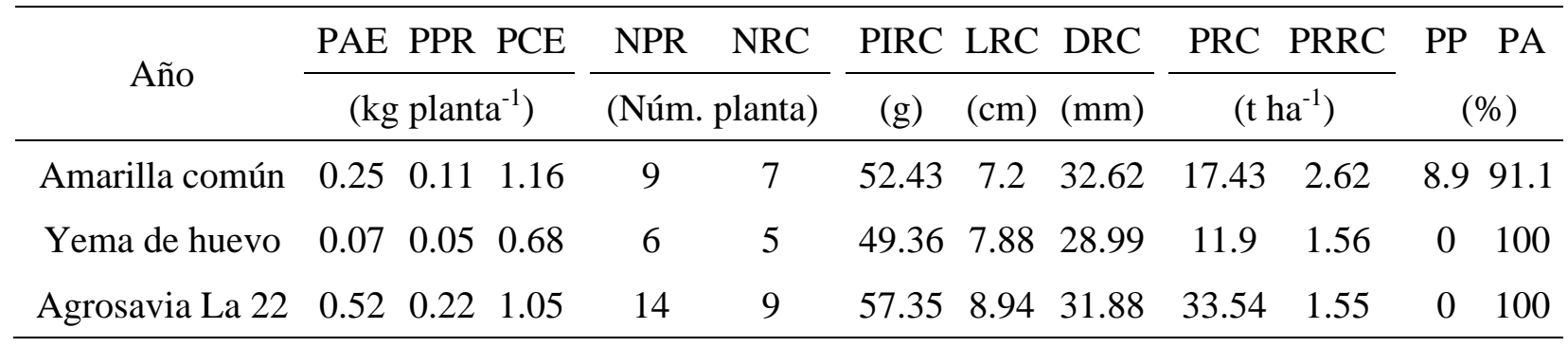

$\mathrm{PAE}=$ peso parte aérea; $\mathrm{PBRO}=$ peso de semilla asexual o propágulo; $\mathrm{PCE}=$ peso de corona o cepa; $\mathrm{NPR}=$ número de propágulos; $\mathrm{NRC}=$ número de raíces comerciales; $\mathrm{PINC}=$ peso individual de raíz tuberosa; $\mathrm{LNC}=$ longitud de raíz tuberosa; $\mathrm{DNC}=$ diámetro de raíz tuberosa, $\mathrm{PRC}=$ peso de raíces comerciales; $\mathrm{PRNC}=$ peso de raíces no comerciales; $\mathrm{PP}=$ porcentaje de raíz tuberosa con pigmentación púrpura; $\mathrm{PA}=$ porcentaje de raíz tuberosa amarilla.

Otra de las características a resaltar de esta nueva variedad de arracacha, es la baja afectación que presentó por plagas y enfermedades. Los niveles de severidad de Alternaria sp., Cercospora sp. y Colletotrichum sp. No superaron en promedio $4.06 \%$ con relación a los materiales regionales, expresando tolerancia a estos fitopatógenos. Con relación a las plagas, se determinó el promedio de individuos adultos de trips y áfidos por planta, estos insectos chupadores generalmente se encuentran en altas poblaciones durante las primeras etapas del cultivo, sin embargo, Agrosavia La 22 presentó menor número de individuos, lo que conllevó a un menor daño por parte de estos, en comparación al resto de los materiales (Cuadro 3). 
Cuadro 3. Severidad (\%) de enfermedades y abundancia de insectos plaga en tres genotipos de arracacha amarilla.

\begin{tabular}{|c|c|c|c|c|c|}
\hline \multirow{2}{*}{ Año } & Alternaria sp. & Colletotrichum sp. & Cercospora sp. & Trips & Áfidos \\
\hline & & $(\%)$ severidad & & \multicolumn{2}{|c|}{ (Núm. planta) } \\
\hline Amarilla común & 6.62 & 1.44 & 0.09 & 36.4 & 20.25 \\
\hline Yema de huevo & 3.81 & 3.34 & 0.62 & 39.76 & 13.77 \\
\hline Agrosavia La 22 & 1.73 & 0.65 & 0.04 & 26.69 & 9.25 \\
\hline
\end{tabular}

De acuerdo con las características de la variedad, se recomienda establecer una densidad de siembra de 20000 plantas ha $^{-1}$ con una distancia de $0.5 \mathrm{~m}$ entre plantas y $1 \mathrm{~m}$ entre surcos y se cosecha entre 9 y 13 meses después de la siembra. Se desarrolla en zonas agroecológicas que presentan altitudes entre los 1800 y 2700 msnm, las cuales tienen un paisaje de montaña en clima frío húmedo y muy húmedo con relieve ondulado ha quebrado y caracterizado por tener suelos derivados de ceniza volcánica, profundos, permeables y textura franco-arenosa. Se adapta en condiciones de temperatura máxima de $27^{\circ} \mathrm{C}$, temperatura mínima de $9.3^{\circ} \mathrm{C}$, temperatura media de $18.1^{\circ} \mathrm{C}$, humedad relativa promedio de $84 \%$, precipitación acumulada de $826.4 \mathrm{~mm}_{\text {año }}{ }^{-1}$ con distribución bimodal, no tolera suelos anegados y requiere muy buena radiación solar.

\section{Descripción de la variedad}

La caracterización cualitativa se realizó teniendo en cuenta el listado de descriptores desarrollado por el Centro Internacional de la Papa [CIP] 2004, seminario (2006) y algunos ajustes de los investigadores de Agrosavia en la especie.

Para identificar la pigmentación de cada órgano se utilizó la tabla de colores Munsell Plant Tissue. Agrosavia La 22 tiene follaje vigoroso con apariencia verde amarilla y hábito de crecimiento erecto e intermedio. La planta posee una altura promedio de $52.97 \mathrm{~cm}$, dosel o canopi de $93.7 \mathrm{~cm}$, longitud de la hoja $23.88 \mathrm{~cm}$, ancho de la hoja $22.66 \mathrm{~cm}$ y 46 peciolos. Las hojas son compuestas con tres o cuatro pares de foliolos alargados y acuminados, tienen coloración verde clara en el haz (5GY4/4, 5GY4/6, 5GY4/8) y verde en el envés (5GY4/6, 5GY4/8, 5GY5/6, 5GY5/8) sin coloraciones secundarias y el borde del foliolo es mediano y profundamente inciso.

El peciolo es cilíndrico, tubular, suculento y hueco, presenta una coloración verde amarilloso (2.5GY6/6, 2.5GY6/8, 2.5GY7/6) con coloración rojiza en la base (5RP3/8, 5RP4/6, 5RP4/8, 5RP3/10, 5RP4/10). La semilla asexual o propágulo es de color rojo-púrpura oscuro (5RP3/6, $5 \mathrm{RP} 3 / 8,5 \mathrm{RP} 3 / 10,5 \mathrm{RP} 4 / 8,5 \mathrm{RP} 4 / 10)$, rosado (5RP5/10) y rojo purpura grisáceo (5RP5/8) con una longitud, diámetro y peso promedio de $5.82 \mathrm{~cm}, 2.91 \mathrm{~cm}$ y $15.25 \mathrm{~g}$ respectivamente, la planta forma 13 propágulos viables.

El tallo es modificado, engrosado y rico en sustancias de reserva. Las raíces tuberosas tienen una longitud promedio horizontal de $21.2 \mathrm{~cm}$, vertical de $12.9 \mathrm{~cm}$ y en total alcanzan hasta $3 \mathrm{~kg}$ de peso, presenta forma cónica e irregular, tiene coloración externa amarilla $(2.5 \mathrm{Y} 8 / 6,2.5 \mathrm{Y} 8 / 4$, $5 \mathrm{Y} 8 / 6)$ sin coloraciones secundarias y la pulpa es $100 \%$ amarilla $(2.5 \mathrm{Y} 8 / 8,5 \mathrm{Y} 8 / 4,5 \mathrm{Y} 8 / 6,5 \mathrm{Y} 8 / 8)$, de forma transversal se evidencia un anillo cortical. Una planta produce en promedio 11 raíces tuberosas de los cuales $81 \%$ es comercial, cada raíz comercial tiene en promedio un diámetro de $5.16 \mathrm{~cm}, 16 \mathrm{~cm}$ de longitud y pesa $266 \mathrm{~g}$. 
La floración en arracacha se evidencia en tres momentos: durante el establecimiento (79 DDS), formación de las raíces tuberosas y después de la cosecha (Garnica-Montaña et al., 2021). En la prueba semicomercial se presentó floración en 3.8\% del total de las plantas en Agrosavia La 22. Una característica no evaluada, pero evidenciada en campo, es la precocidad de la planta puesto que la cosecha es posible entre 30 y 60 días antes de los 12 meses, lo cual se debe corroborar con mayor investigación.

Las características previas muestran que Agrosavia La 22 es un material de arracacha con potencial para posicionar el sistema productivo, sus altos rendimientos, tonalidad completamente amarilla y mayor porcentaje de raíces comerciales podrían generar solución a las demandas y necesidades de los productores colombianos (Figura 1).

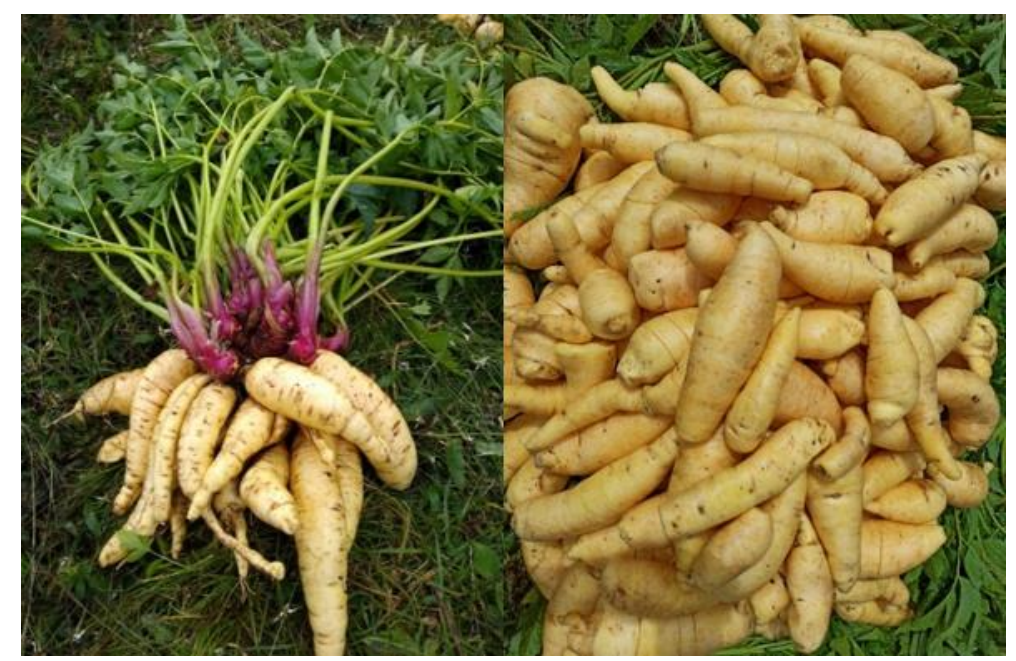

Figura 1. a) planta de variedad Agrosavia La 22 y b) raíces tuberosas comerciales.

\section{Conclusiones}

Se registró la primera variedad de arracacha en Colombia, amarilla, de alta producción y adaptada a condiciones agroecológicas de la zona andina que superó $40 \%$ el rendimiento de los cultivares testigos, los atributos de la nueva variedad son: pigmentación de pulpa de raíces tuberosas $100 \%$ amarilla, $81 \%$ de raíces tuberosas comerciales y con porcentajes que no superan $2.5 \%$ de afectación por enfermedades foliares ocasionadas por Colletotrichum sp., Alternaria sp. y Cercospora sp.

\section{Agradecimientos}

Al Sistema de Bancos de Germoplasma de la Nación para la Alimentación y la Agricultura (SBGNAA) como fuente del material biológico. Esta investigación se desarrolló en el macroproyecto 'Generación y vinculación de tecnologías para mejorar la productividad de los sistemas de producción de arracacha en Colombia'. Proyectos: una variedad de arracacha amarilla seleccionada por adaptación y productividad y Nuevos materiales de siembra, uso de diversidad genética y propagación de genotipos de arracacha, financiados por la Corporación Colombiana de Investigación Agropecuaria-AGROSAVIA. A los investigadores promotores de esta gran iniciativa de los doctores Juan José Rivera Varón y Jorge Alberto Valencia Montoya por su contribución técnica, operativa y administrativa. 


\section{Literatura citada}

Albano, K. M.; Franco, C. M. L. and Telis, V. R. N. 2014. Rheological behavior of Peruvian carrot starch gels as affected by temperature and concentration. Food Hydrocolloids. 40:30-43. https://doi.org/10.1016/j.foodhyd.2014.02.003.

Alvarado, Á. and Ochoa, L. 2010. Tecnologías locales de producción de arracacha (Arracacia xanthorrhiza Bancroft) en el municipio de Boyacá, departamento de Boyacá. Rev. UDCA Act. \& Div. Cient, 13(1):125-133. https://doi.org/10.1371/journal.pone.0152157.

Bell, R. and Constance, L. 1966. Chromosome numbers in umbelliferae. III. Am. J. Bot. 53(5):512520. https://doi.org/10.1002/j.1537-2197.1966.tb07365.x.

CARC. 2014. Cooperativa Multiactiva Productores Agricolas Cajamarca. Ficha técnica de selección de arracacha.

CORTOLIMA. 2006. Corporación Autónoma Regional del Tolima. Plan de ordenación y manejo de la cuenca hidrográfica mayor del río Coello.

Dos Santos, F. 2004. Producción de arracacha en Brasil. In: Seminario, J. (Ed.). Raíces andinas, contribuciones al conocimiento y a la capacitación. $6 \mathrm{p}$.

Garnica-Montaña, J. P.; Villamil-Carvajal, J. E.; Vargas-Berdugo, A. M. y Rodríguez-Rodríguez, O. J. 2021. Modelo productivo para la producción de la primera variedad de arracacha (Arracacia xanthorrhiza Bancr.) Agrosavia La 22 adaptada a la región natural Andina de Colombia. Corporación Colombiana de Investigación Agropecuaria (AGROSAVIA). Mosquera, Colombia. 127 p. https://doi.org/10.21930/agrosavia.nbook.7404500.

Hermann, M. 1997. Arracacha. Arracacia xanthorrhiza Bancroft. In: Hermann, M. and Heller, J. (Ed.), Andean roots and tubers: ahipa, arracacha, maca and yacon Institute of Plant Genetics and Crop Plant Research, Gatersleben/International Plant Genetic Resources Institute. https://doi.org/10.1371/journal.pcbi.0030059.eor. 75-172 pp.

Knudsen, S. 2003. Reproduction biology of the Andean root crop arracacha (Arracacia xanthorrhiza Bancroft var. xanthorrhiza) and the taxonomic status of the South American Arracacia Bancroft species with special emphasis on the position of the cultivated arracacha and rela. The Royal Veterinary and Agricultural University. Universidad de Copenhague. 150 p.

Leidi, E. O.; Monteros-Altamirano, A.; Mercado, G.; Rodriguez, J. P.; Ramos, A.; Alandia, G., Sorensen, M. y Jacobsen, S. E. 2018. Andean roots and tubers crops as sources of functional foods. J. Functional Foods. 51:86-93. https://doi.org/10.1016/j.jff.2018.10.007.

Madeira, N. y Ferreira, A. 2016. Produção de mudas de mandioquinha-salsa. In: Nascimento, W. y Borges, R. (Ed.). Hortalicas de propagacao vegetativa, tecnologia de multiplicacao. Embrapa. 129-150 pp.

MINTIC. 2018. Ministerio de Tecnologías de la Información y las Comunicaciones-Evaluaciones Agropecuarias Municipales EVA. Portal de Datos Abiertos www.datos.gov.co. https://www.datos.gov.co/Agricultura-y-Desarrollo-Rural/Evaluaciones-AgropecuariasMunicipales-EVA/2pnw-mmge/data.

Morillo, E.; Second, G.; Pham, J. L. and Risterucci, A. M. 2004. Development of DNA microsatellite markers in the Andean root crop arracacha: Arracacia xanthorrhiza Banc. (Apiaceae). Mol. Ecol. Notes. 4:680-682. https://doi.org/10.1111/j.14718286.2004.00783.x.

Muñoz, A.; Alvarado, A. y Almanza-Merchán, P. 2015. Caracterización preliminar del cultivo de arracacha Arracacia xanthorrhiza Bancroft en el departamento de Boyacá. Rev. Cienc. Agríc. 32(1):3-11. 
Pinto-Acero, Y. L.; Alvarado-Gaona, Á. E.; Burgos-Ávila, Y. E.; Balaguera-lópez, H. E. y Ramírez-Gonzáñez, S. I. 2019. Characterization of three Arracacia xanthorrhiza Bancroft genotypes using morphological and color parameters Caracterización de tres genotipos de Arracacia xanthorrhiza Bancroft mediante parámetros morfológicos y de color. Rev. Colomb. Cienc. Hortíc. 13(3):426-434. https://doi.org/https://doi.org/10.17584/ rcch.2019v13i3.8948.

Rueda, L. 1992. Andean roots and tubers. In: Posner, J. (Ed.). The consortium for sustainable development in the Andean ecoregion (CONDESAN). 1-21 p.

Scott, G. J.; Rosegrant, M. y Ringler, C. 2000. Raíces y tubérculos para el Siglo 21 tendencias, proyecciones y opciones de política. Instituto Internacional de Investigaciones sobre Políticas Alimentarias (IFPRI) (Ed.). Washington, EE.UU. 72 p.

Vasquez, N.; Medina, C. y Lobo, M. 2004. Caracterización morfológica de la colección colombiana (Tolima, Huila, Boyacá, Cauca) de arracacha (Arracacia xanthorrhiza). In: J. seminario (Ed.). Raíces Andinas, contribuciones al conocimiento y a la capacitación. 14 p. 